\title{
CONTRIBUIÇÃO PALINOLÓGICA AO CONHECIMENTO DO SUBGRUPO ITARARÉ NA SERRA DOS PAES, SUL DO ESTADO DE SÃO PAULO
}

\author{
Paulo Alves de SOUZA \\ Fernando Farias VESELY \\ Mario Luis ASSINE
}

\author{
RESUMO
}

\begin{abstract}
Este trabalho constitui a primeira contribuição paleontológica para o Subgrupo Itararé na Serra dos Paes, sul do Estado de São Paulo, correspondendo à porção superior da unidade na área. Quinze táxons foram reconhecidos, dos quais dois são inéditos para a porção brasileira da Bacia do Paraná: Granulatisporites austroamericanus Archangelsky \& Gamerro e Protohaploxypinus panaki Utting. A assembléia é dominada por grãos de pólen teniados e poliplicados, destacando-se os gêneros Protohaploxypinus, Vittatina e Complexisporites, enquanto que esporos e grãos de pólen não-teniados são raros. Considerando o arcabouço palinobioestratigráfico da Bacia do Paraná e das bacias gondvânicas circunvizinhas, a idade da assembléia é considerada eopermiana (Sakmariano).

Palavras-chave: Subgrupo Itararé, palinologia, Serra dos Paes, Permiano, Bacia do
\end{abstract} Paraná.

\section{ABSTRACT}

Fifteen sporomorphs have been recorded from the upper portion of the Itarare Subgroup, Paraná Basin, at Serra dos Paes, south of São Paulo State, Brazil. This represents the first palynological record for the locality. The palynological assemblage is rich in taeniate bisaccate and poliplicat pollen grains such as Protohaploxypinus, Vittatina and Complexisporites, whereas spores and non-taeniate (mono and bisaccate) pollen grains are scarce. Two taxa are presented for the first time in the Brazilian Paraná Basin: Granulatisporites austroamericanus Archangelsky \& Gamerro and Protohaploxypinus panaki Utting. According to the palynological data and to correlations with similar Gondwanic assemblages, the age of the assemblage is considered to be early Early Permian (Sakmarian).

Keywords: Itararé Subgroup, palynology, Serra dos Paes, Permian, Paraná Basin.

\section{INTRODUÇÃO}

O Subgrupo Itararé é uma unidade litoestratigráfica da Bacia do Paraná depositada no intervalo neocarbonífero/eopermiano. Compreende diversos litotipos (arenitos, diamictitos, conglomerados, ritmitos, entre outros) que ocorrem da base até o topo com alto grau de recorrência. Há grande variação lateral de fácies, sendo difícil a caracterização de camadas-guia, importantes para a correlação estratigráfica e análise de seqüências.

Por essa razão, os dados palinológicos fornecem subsídios para correlação estratigráfica e determinação da idade dos diferentes intervalos. Merece destaque o zoneamento de DAEMON \& QUADROS (1970), o mais abrangente em termos geográficos e estratigráficos já realizado na Bacia do Paraná.

A sucessão estratigráfica do Subgrupo Itararé na região da Serra dos Paes pertence à parte superior da unidade (CAETANO-CHANG 1984, CAETANO-CHANG \& LANDIM 1987, CABRAL JR. et al. 1988). À exceção da ocorrência de invertebrados na localidade vizinha de Itaporanga (MEZZALIRA 1956), não há nenhuma outra referência paleontológica na região. 
Os dados palinológicos aqui apresentados constituem informações inéditas e visam a contribuir para melhor conhecimento da sucessão palinológica e posicionamento cronoestratigráfico da unidade.

\section{MATERIAIS E MÉTODOS}

A Serra dos Paes está localizada na faixa de afloramentos da margem oriental da Bacia do Paraná, no flanco norte do Arco de Ponta Grossa (Figura 1). Nela estão expostos cerca de $150 \mathrm{~m}$ da seção superior do Subgrupo Itararé, capeados por litologias do Subgrupo Guatá (Formação Tatuí).

Foram amostrados diamictitos maciços cinza-esverdeados posicionados próximos ao topo do Subgrupo Itararé. O ponto de coleta situa-se a 5 quilômetros ao norte da cidade de Barão de
Antonina, nas coordenadas geográficas $23^{\circ} 35^{\prime}$ de latitude Sul e 49³4' de longitude Oeste.

A amostra coletada foi desagregada fisicamente (maceração), com posterior dissolução de seus componentes inorgânicos (reação com $\mathrm{HCl}, \mathrm{HF})$ e minerais neoformados $(\mathrm{HCl}$ à quente) e concentração final do resíduo na fração entre $0,25-0,025 \mathrm{~mm}$. A partir do resíduo palinológico, foram confeccionadas três lâminas, codificadas e depositadas na Palinoteca do Instituto Geológico/SMA, sob números IG-P: 291A, B e C.

Neste trabalho, são apresentados os principais resultados da análise qualitativa referente a cinco espécies de esporos e dez de grãos de pólen. Em virtude de o material ter se mostrado bastante escasso, não foi possível ser realizada análise quantitativa, para fins de representatividade paleobotânica e interpretação paleoambiental.

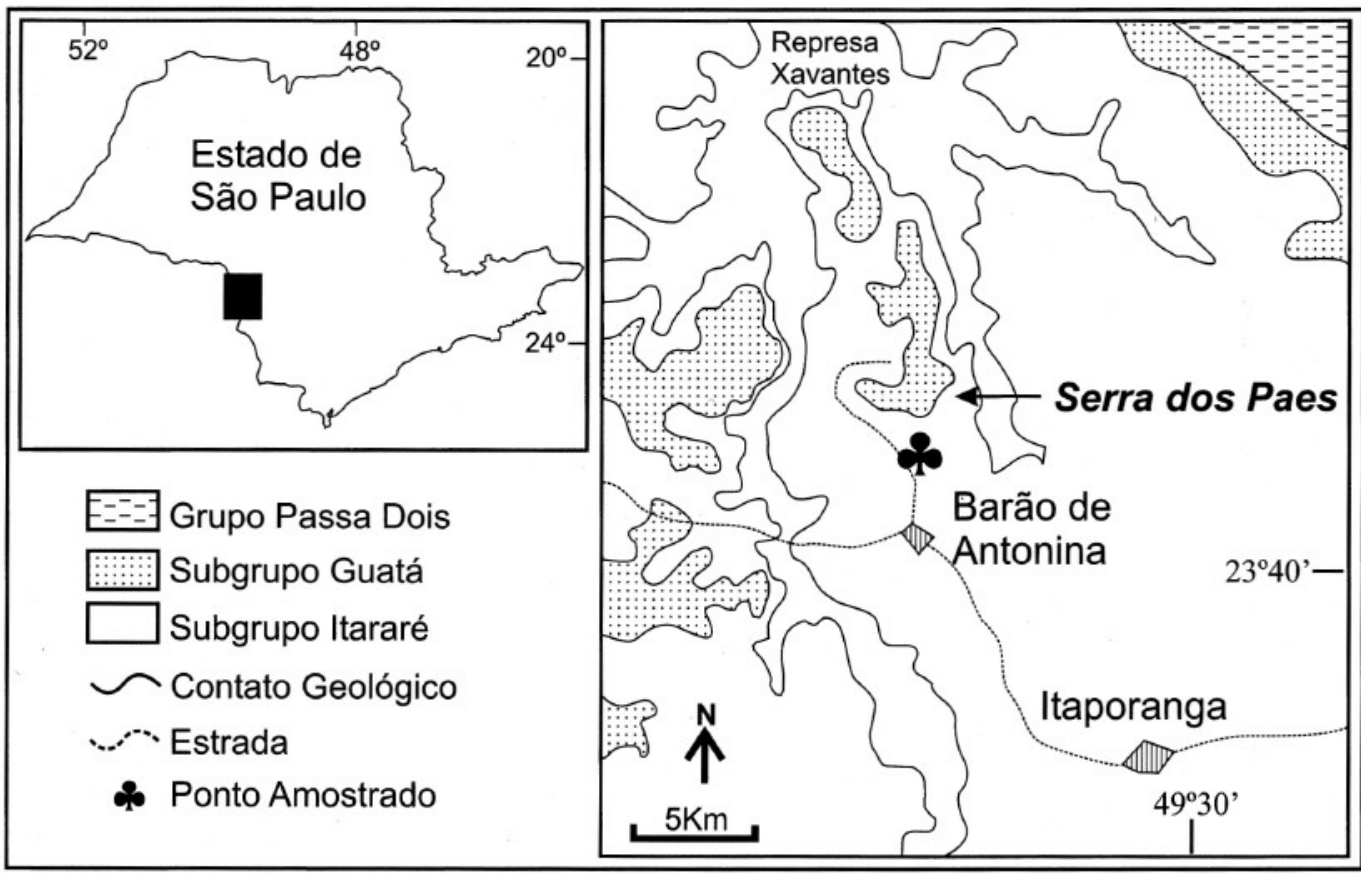

FIGURA 1 - Situação geológica geral da área e localização da amostragem palinológica.

\section{RESULTADOS PALINOLÓGICOS}

A composição palinológica verificada é marcada pela variedade de grãos de pólen teniados, em detrimentos dos esporos e grãos de pólen monossacados e bissacados não-teniados. Essas características são típicas das assembléias palinológicas do limite Carbonífero/Permiano e de todo o Permiano Inferior das bacias gondvânicas, como já registrado na Bacia do Paraná (ARAI 1980, PICARELLI et al. 1987).

A seguir são relacionados os principais palinomorfos reconhecidos, alguns dos quais identificados em nível genérico. Destaca-se que duas espécies são noticiadas pela primeira vez 
na Bacia do Paraná: Granulatisporites austroamericanus e Protohaploxypinus panaki.

Esporos: Calamospora hartungiana Schopf, Wilson \& Bentall, 1944; Granulatisporites austroamericanus Archangelsky \& Gamerro, 1979; Horriditriletes uruguaiensis (MarquesToigo) Archangelsky \& Gamerro, 1979; Cristatisporites inconstans Archangelsky \& Gamerro, 1979; Vallatisporites spp.

Grãos de pólen: Cannanoropollis sp.;

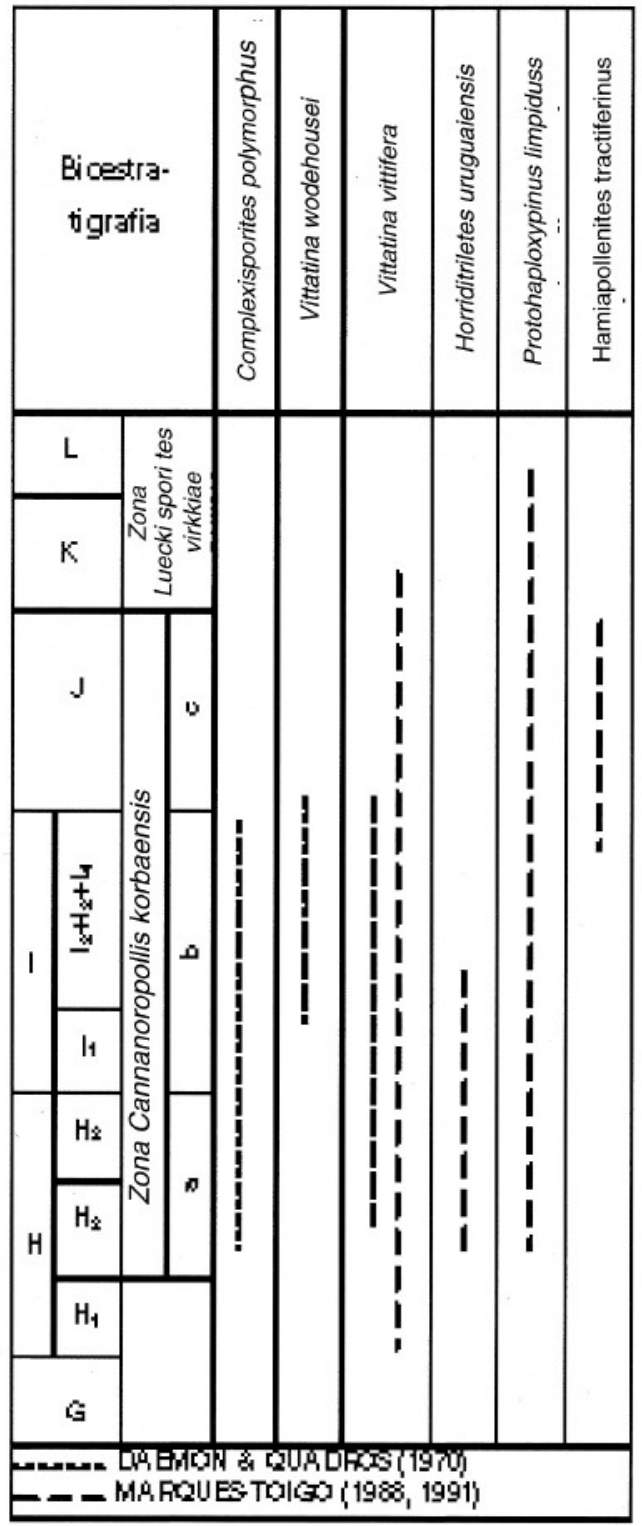

QUADRO 1 - Distribuição de algumas espécies verificadas de acordo com os zoneamentos palinobioestratigráficos da Bacia do Paraná (a: Subzona Protohaploxypinus-goraiensis, b: Subzona Caheniasaccites ovatus, c: Subzona Hamiapollenites karroensis).
Caheniasaccites sp.; Protohaploxypinus limpidus (Balme \& Hennelly) Balme \& Playford, 1968; Protohaploxypinus micros (Hart) Hart, 1964; Protohaploxypinus panaki Utting, 1994; Striatoabieites sp.; Hamiapollenites tractiferinus (Samoilovich) Hart, 1964; Complexisporites polymorphus Jizba, 1964; Vittatina wodehousei (Jansonius) Hart, 1964; Vittatina vittifera (Lüber \& Valtz) Samoilovich, 1953.

\section{DISCUSSÃO}

Das formas reconhecidas, apenas três integram parte do zoneamento de DAEMON \& QUADROS (1970): Complexisporites polymorphus (forma P425), Vittatina wodehousei (P386), e $V$. vittifera (P392). Do zoneamento de MARQUES-TOIGO (1988), estabelecido para a seqüência neopaleozóica da bacia nos estados do Rio Grande do Sul e Santa Catarina, quatro espécies são comuns: Horriditriletes uruguaiensis, Protohaploxypinus limpidus, Hamiapollenites tractiferinus e Vittatina vittifera.

No Quadro 1 são apresentadas as distribuições dos táxons comuns constantes nos zoneamentos de DAEMON \& QUADROS (1970), MARQUES-TOIGO (1988). Foi possível identificar convergência de resultados para o intervalo $\mathrm{H}_{3}-\mathrm{I}$, correspondente às subzonas Protohaploxypinus goraiensis e Caheniasaccites ovatus, das posições inferior e média da Zona Cannanoropolis korbaensis. Algumas divergências são verificadas com relação à distribuição de espécies no zoneamento estabelecido por MARQUES-TOIGO (1988) para os estados de Santa Catarina e Rio Grande do Sul, o que pode ser conseqüência de diferente amplitude dos táxons nas regiões mais meridionais da bacia.

Comparações com assembléias palinológicas de outras bacias gondvânicas da América do Sul sugerem idade eopermiana para os diamictitos da parte superior do Subgrupo Itararé na Serra dos Paes.

As espécies Granulatisporites austroamericanus, Horriditriletes uruguaiensis, Cristatisporites inconstans e Protohaploxypinus micros têm ocorrência comum nas zonas Potonieisporites-Lundbladispora e Cristatisporites inferior da Bacia Chacoparaná na Argentina e correspondentes no Uruguai (ARCHANGELSKY \& GAMERRO 1979, 
BERI 1988, VERGEL 1993, MAUTINO et al. 1998), posicionadas na base do Eopermiano.

Granulatisporites austroamericanus e Protohaploxypinus panaki, espécies noticiadas pela primeira na Bacia do Paraná, foram descritas no Permiano Inferior da Argentina e do Canadá, respectivamente.

Esse posicionamento cronoestratigráfico é divergente daquele proposto por DAEMON \& QUADROS (1970) para os mesmos intervalos bioestratigráficos da Bacia do Paraná, segundo o qual o intervalo $\mathrm{H}_{3}-\mathrm{I}_{4}$ seria relativo ao Artinskiano/Kunguriano (topo do Eopermiano). Como já expressado por SOUZA (1996), há a necessidade de uma reavaliação das idades dos intervalos bioestratigráficos em razão de divergências com resultados provenientes de outros grupos fósseis da Bacia do Paraná (e.g. macrofitofósseis: RÖSLER 1978) e com as idades propostas para os zoneamentos palinológicos das bacias gondvânicas circunvizinhas.

O conjunto palinológico descrito apresenta espécies comuns e características gerais semelhantes às assembléias palinológicas identificadas na parte superior do Subgrupo Itararé no Estado de São Paulo, mais precisamente no carvão de Cerquilho, posicionado no intervalo $\mathrm{I}_{2}-\mathrm{I}_{4}$ (SOUZA et al. 1993), e na Formação Rio do Sul aflorante nos estados do Paraná e de Santa Catarina, cujos estratos foram posicionados no intervalo $\mathrm{H}_{3}-\mathrm{I}_{4}$ (CANUTO 1985).

Em subsuperfície, nos poços J-IG-93, C-IG93, T-IG-91, T-IG-93 (perfurados pelo IGSMA), 2-CB-1-SP, 2-TB-1-SP, 2-PN-1-SP, 1RO-1-PR (Paulipetro) e 1-SJ-1-PR (Petrobrás), as correlações bioestratigráficas são com as partes superior do Subgrupo Itararé (Formação Taciba) e basal das formações Tatuí e Rio Bonito nos estados de São Paulo e Paraná.

Considerando a amplitude dos táxons e, em maior escala, a própria distribuição dos intervalos palinobioestratigráficos, verifica-se que os dados palinológicos disponíveis não permitem a diferenciação das duas unidades litoestratigráficas. A maioria dos táxons descritos em litologias do Subgrupo Itararé na Serra dos Paes ocorre também na base da Formação Rio Bonito em outras localidades.

$\mathrm{Na}$ área de Ribeirão Novo, município de Wenceslau Brás (PR), situada a $60 \mathrm{~km}$ a sudoeste da Serra dos Paes, arenitos com carvão são recobertos por $50 \mathrm{~m}$ de diamictitos. Este intervalo foi posicionado por MARQUES-TOIGO et al. (1981) no Membro
Triunfo da Formação Rio Bonito, onde reconheceram dois níveis carbonosos, com conteúdo palinológico distinto entre si, relacionados a associações paleobotânicas higrófilas e hidrófilas. Embora possa ser cronocorrelata à seção analisada na Serra dos Paes, não é possível realizar qualquer correlação palinológica, tendo em vista o tratamento sistemático em nível genérico e a ausência de grãos de pólen teniados naqueles níveis.

\section{CONCLUSÕES}

1) Diamictitos presentes no topo do Subgrupo Itararé na Serra dos Paes, município de Barão de Antonina (SP), são portadores de palinomorfos das subzonas Protohaploxypinusgoraiensis e Caheniasaccites ovatus da Zona Cannanoropolis korbaensis, correspondente aos intervalos $\mathrm{H}_{3}-\mathrm{I}_{4}$.

2) Por correlação com outros estratos gondvânicos, a idade mais indicada para a associação é base do Eopermiano (Sakmariano).

3) O posicionamento da parte superior do Subgrupo Itararé na base do Eopermiano, a partir de correlação com zoneamentos mais atualizados existentes em outras bacias gondvânicas, revela a necessidade da reavaliação cronoestratigráfica do zoneamento palinobioestratigráfico da Bacia do Paraná.

4) Se confirmada em outras áreas a idade obtida para o topo do Subgrupo Itararé, configura-se a necessidade de se "baixar" as idades de várias unidades e eventos permo-carboníferos da Bacia do Paraná, a começar por se considerar que a glaciação começou e terminou antes, tendo transcorrido, sobretudo, no Neocarbonífero.

\section{AGRADECIMENTOS}

Os autores agradecem o apoio da FAPESP à realização deste trabalho, desenvolvido no âmbito dos projetos "Levantamento da composição e sucessão paleoflorísticas do Neocarbonífero-Eopermiano (Grupo Tubarão) do Estado de São Paulo" (processo $\mathrm{n}^{\circ}$ 97/03639-8) e "Análise de paleocorrentes e paleogeografia da Bacia do Paraná" (processo ${ }^{\circ}$ 98/02183-3). 


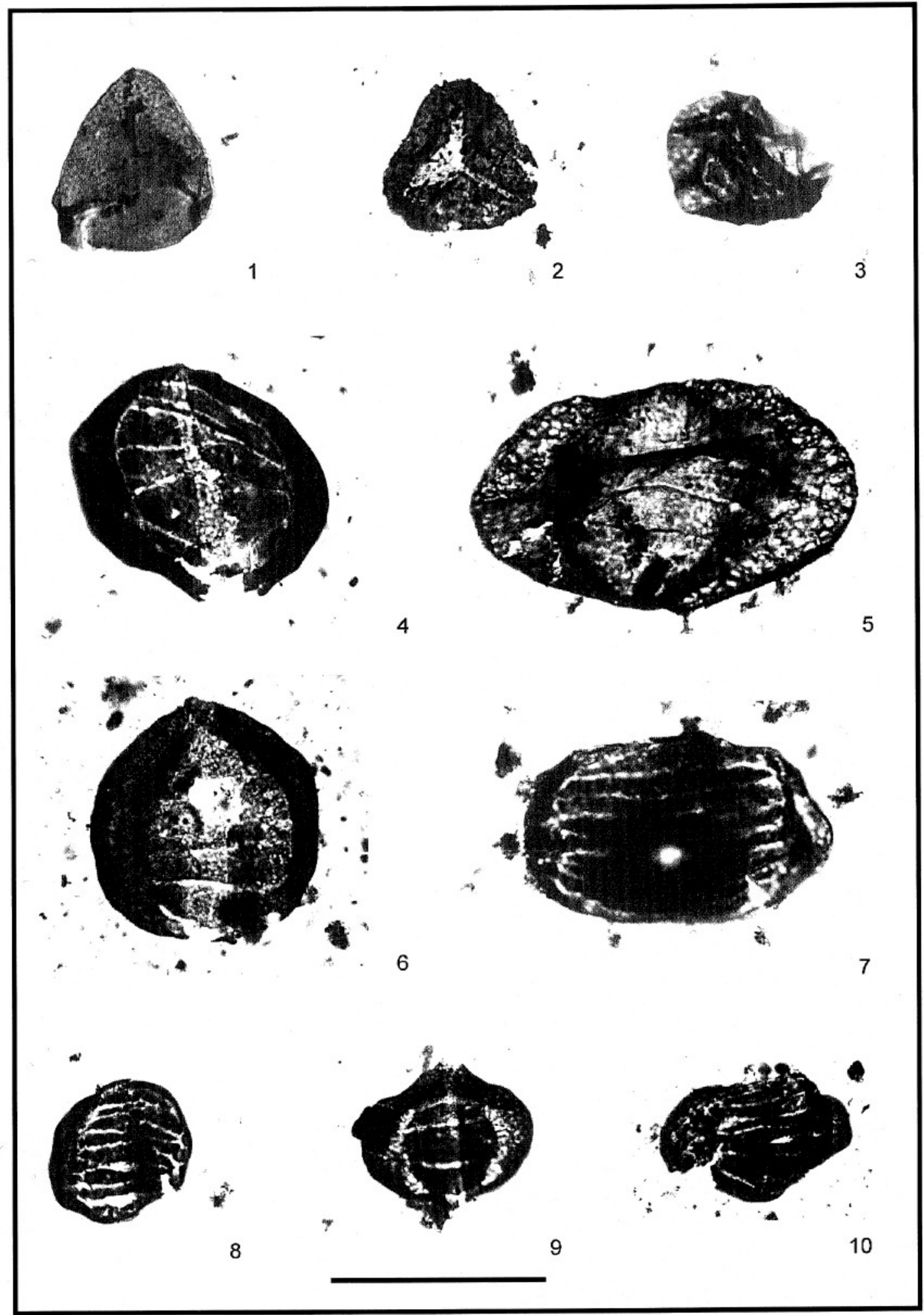

ESTAMPA 1 - Fig. 1. Granulatisporites austroamericanus (lâmina IG-P: 291A, coordenada England Finder

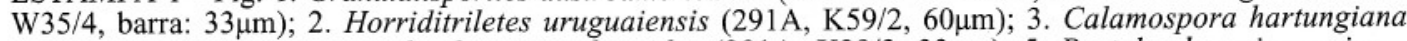

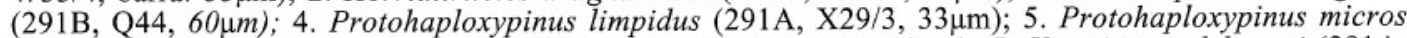

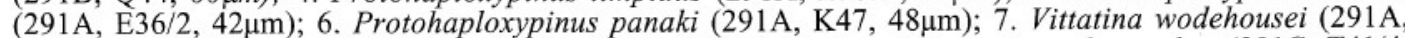
$\mathrm{M} 45 / 3,42 \mu \mathrm{m}) ; 8$. Vittatina vittifera $(291 \mathrm{~B}, \mathrm{~T} 27 / 4,50 \mu \mathrm{m}) ; 9$. Complexisporites polymorphus $(291 \mathrm{C}, \mathrm{T} 41 / 4$, $58 \mu \mathrm{m}) ; 10$. Hamiapollenites tractiferinus (291A, K47/4, 66 $\mu \mathrm{m})$. 


\section{REFERÊNCIAS BIBLIOGRÁFICAS}

ARAI, M. 1980. Contribuição dos pólens estriados na bioestratigrafia neopaleozóica da parte nordeste da Bacia do Paraná. São Paulo: Boletim IG-USP, 11:98-105.

ARCHANGELSKY, S. \& GAMERRO, J.C. 1979. Palinologia del Paleozoico Superior en el subsuelo de la Cuenca Chacoparanense, República Argentina. I. Estudio sistemático de los palinomorfos de tres perfaciones de la Província de Córdoba. Madrid: Revista Española de Micropaleontología, XI(3):417-478.

BERI, A. 1988. Estudio sistematico del contenido palinológico de una muestra de la Formación Tres Islas (Pérmico Inferior) del Uruguay. Porto Alegre: PaulaCoutiana, 3:27-48.

CABRAL JR., M.; CAMPANHA, V.A.; MOTTA, J.F.M. ; SAAD, A.R. 1988. Contribuição à estratigrafia e paleogeografia da interface Itararé e Tatuí $(\mathrm{P})$ e considerações sobre sua potencialidade para carvão no Estado de São Paulo. In: SBG, CONGRESSO BRASILEIRO DE GEOLOGIA, XXXV, Belém, Anais, 2:879-893.

CAETANO-CHANG, M.R. 1984. Análise ambiental e estratigráfica do Subgrupo Itararé (PC) no sudoeste do Estado de São Paulo. Instituto de Geociências, Universidade de São Paulo, São Paulo, Tese de Doutoramento, 309p.

\& LANDIM, P.M.B. 1987. Evolução ambiental da sedimentação do Subgrupo Itararé na área de ItararéItaporanga (SP). In: SBG/SP, SIMPÓSIO REGIONAL DE GEOLOGIA, $6^{\circ}$, Rio Claro, Atas, 1:339-354.

CANUTO, J.R. 1985. Origem dos diamictitos e de rochas associadas do Subgrupo Itararé, no sul do Estado do Paraná e norte do Estado de Santa Catarina. Instituto de Geociências, Universidade de São Paulo, São Paulo, Dissertação de Mestrado, 187p.

DAEMON, R. F. \& QUADROS, L.P. 1970. Bioestratigrafia do Neopaleozóico da Bacia do Paraná. In: SBG, CONGRESSO BRASILEIRO DE GEOLOGIA, 24, Brasília, Anais, p. 359-412.

MARQUES TOIGO, M. 1988. Palinologia, bioestratigrafia e paleoecologia do
Neopaleozóico da Bacia do Paraná nos estados do Rio Grande do Sul e Santa Catarina, Brasil. Instituto de Geociências, Universidade Federal do Rio Grande do Sul, Porto Alegre, Tese de Doutoramento, 259p., 9est.

DIAS-FABRÍCIO, M.E.; KLEPZIG, M.C.; OLIVEIRA, L.M. ; DAVID, C.A.S. 1981. Caracterização palinológica de camadas de carvão da área de Ribeirão Novo (PR), Paleozóico da Bacia do Paraná-Brasil. In: SBG, SIMPÓSIO REGIONAL DE GEOLOGIA, $3^{\circ}$, Curitiba, Atas, 2:310-321.

MAUTINO, L.R.; VERGEL, M.M. ; ANZÓTEGUI, L.M. 1998. Palinología de la Formación Melo (Pérmico Inferior) en Arroyo Seco, Departamento Rivera, Uruguay. Parte V: granos de polen, acritarcas e incertae sedis. Buenos Aires: Ameghiniana, 35(3):299-314.

MEZZALIRA, S. 1956. Novas ocorrências de camadas marinhas permo-carboníferas no Estado de São Paulo. São Paulo: Boletim da Sociedade Brasileira de Geologia, 5(1):61-69.

PICARELLI, A.T.; DIAS-FABRÍCIO, M.E. ; CAZZULO-KLEPZIG, M. 1987. Considerações sobre a paleoecologia e a palinologia da jazida carbonífera de Santa Terezinha, RS, Brasil - Permiano da Bacia do Paraná. In: SBG, SIMPÓSIO SULBRASILEIRO DE GEOLOGIA, III, Curitiba, Atas, 1:351-372.

RÖSLER, O. 1978. The Brazilian eogondwanic floral sucessions. São Paulo: Boletim IGUSP, 9:85-91.

SOUZA, P.A. 1996. Palinologia e bioestratigrafia do Subgrupo Itararé em Araçoiaba da Serra (Westphaliano, Bacia do Paraná), Estado de São Paulo, Brasil. Instituto de Geociências, Universidade de São Paulo, São Paulo, Dissertação de Mestrado, 192p., 12est.

; LIMA, M.R. de ; SAAD, A.R. 1993. Palinologia dos carvões paleozóicos do Estado de São Paulo. III-O Carvão de Cerquilho. In: SBP, CONGRESSO BRASILEIRO DE PALEONTOLOGIA, $13^{\circ}$, São Leopoldo, Resumos, p. 62. 
VERGEL. M.M. 1993. Palinoestratigrafía de la secuencia neopaleozóica en la Cuenca Chacoparanense, Argentina. In: INTER-
NATIONAL CONGRES ON CARBONIFEROUS-PERMIAN, XII, Buenos Aires, Comptes Rendus, 1:201-212.

Endereço dos autores:

Paulo Alves de Souza - Instituto Geológico/SMA, Av. Miguel Stéfano, 3.900, CEP 04301-903, São Paulo, SP, Brasil. E-mail: psouza@ igeologico.sp.gov.br

Fernando Farias Vesely - IGCE/UNESP, Pós-Graduação, Av. 24-A, 1.515, CEP 13506-900, Rio Claro, SP, Brasil. E-mail: ffvesely@ rc.unesp.br

Mario Luis Assine - IGCE/UNESP, Departamento de Geologia Aplicada, Av. 24-A, 1.515, CEP 13506-900, Rio Claro, SP, Brasil. E-mail: assine @ rc.unesp.br 